\title{
Passive Flow Control over an Airfoil by Control Rod at Low Reynolds Number
}

\author{
T. Durhasan \\ Aerospace Engineering Department, Adana Alparslan Turkes Science and Technology University, Adana, \\ 01250, Turkey
}

†Corresponding Author Email: tdurhasan@atu.edu.tr

(Received 23 October, 2019; accepted April 4, 2020)

\begin{abstract}
In the present study, the flow control mechanism of SD7062 airfoil by a rod illustrated using Particle image velocimetry (PIV) technique at pre-stall angles of attack at Reynolds number of $R e=30000$. The rod was installed on the suction surface of the airfoil at different chordwise locations. Diameter of the rod was normalized with the chord length of the airfoil and three diameter ratios $(\mathrm{d} / \mathrm{c}=0.017,0.033$ and 0.044$)$ were examined at angles of attack of $\alpha=6^{\circ}, 8^{\circ}$ and $10^{\circ}$. Formation of laminar separation bubble for the baseline airfoil and the effect of rod on the laminar separation bubble were investigated in detail. It is observed that the height of boundary layer was reduced up to $22 \%$ by proper rod location and diameter ratio. Moreover, the rod suppressed the unsteady vortices over the suction surface of airfoil significantly. Therefore, the peak magnitudes of turbulent statistics were also decreased up to $30 \%$ by the rod.
\end{abstract}

Keywords: Airfoil; Control rod; Flow control; Low Reynolds number flow.

\section{NOMENCLATURE}

$\begin{array}{ll}\mathrm{c} & \text { chord length of airfoil } \\ \mathrm{d} & \text { diameter of rod } \\ \mathrm{d} / \mathrm{c} & \text { diameter ratio } \\ \text { LSB } & \text { laminar separation bubble } \\ \text { PSD } & \text { power spectra density } \\ \mathrm{Re} & \text { Reynolds number } \\ \mathrm{s} & \text { span length of airfoil } \\ \mathrm{St} & \text { Strouhal number } \\ \text { TKE } & \text { turbulent kinetic energy } \\ \mathrm{U}_{\infty} & \text { free stream velocity }\end{array}$

\section{INTRODUCTION}

Low Reynolds flow is encountered commonly in many engineering applications such as micro air vehicles, small-scale wind turbines, small scalehydro turbines, etc. The most distinct flow characteristic in the low Reynolds number applications is formation of laminar separation bubble (LSB) and it may negatively affect the flow behavior and aerodynamic performance such as vibration, enhancing of the drag and reducing of the lift. Formation of LSB and its effect on the aerodynamic performance are discussed in detail by O'Meara and Mueller (1987); Rinoie and Takemura (2004); Burgmann et al. (2006); Zhang et al. (2008). Flow control around airfoil have been

$\begin{array}{ll}\mathrm{u}_{\mathrm{rms}} & \begin{array}{l}\text { root mean square of streamwise velocity } \\ \text { mean streamwise velocity } \\ u^{y} v^{y}\end{array} \\ \mathrm{x} / \mathrm{c} & \begin{array}{l}\text { time averaged Reynolds shear stress } \\ \text { chordwise section }\end{array} \\ \alpha & \begin{array}{l}\text { angle of attack } \\ \text { vorticity }\end{array} \\ \bar{\Psi} & \text { time averaged streamline topology }\end{array}$

applied to not only suppress of adverse effect of LSB but also enhance the aerodynamic performance of the airfoil, therefore, various flow control techniques classified into two main groups as active and passive have been studying. Although the active flow control techniques such as suction or blowing (Huang et al. 2004; Yousefi and Saleh 2014), acoustic noise (Ricci et al. 2007; Genç et al. 2016), dielectric barrier discharge plasma actuators (Tathiri et al. 2016; Khoshkhoo and Jahangirian 2016; Akbiylk et al. 2017) are highly successful in the suppressing of adverse effect of the flow behavior over airfoil or enhancing of aerodynamic performance, their applications require high cost devices and external energy. On the other hand, implementation of passive flow control techniques is relatively simple and inexpensive. Moreover, 
they have a broad application area such as micro unmanned aerial vehicles since some passive flow control techniques do not generate weight, volume and require power consumption.

Lee and Jang (2005) experimentally studied flow over NACA 0012 airfoil with the micro riblet surface at Reynolds number in the range of 10300 $\leqslant \mathrm{Re} \leqslant 51400$. They indicated that riblet surface reduces the drag coefficient by $6.6 \%$ at $\mathrm{Re}=10300$ due to the decreasing of spanwise momentum transport and the vortex formation region. However, the drag coefficient increases at $\mathrm{Re}=51400$, since riblet surface leads to formation of small scale vortices and increasing of vortex formation region. Sefiddashti et al. (2018) experimentally examined the effect of riblets on the drag reduction of Ris $\varnothing$ airfoil. They indicated that the riblet valleys reduces the shear stress near the airfoil surface and found that at the angle of attack $\alpha=7^{\circ}$, the drag coefficient is reduced by $29.7 \%$ and $54 \%$ at $\mathrm{Re}=$ 202000 and 140000, respectively. Rinoie et al. (2009) attached a thin plate near the leading edge of NACA 0012 airfoil to control laminar separation bubble at $\mathrm{Re}=130000$ and found that thin plate causes the formation of short bubble and stall is suppressed remarkably because of the reattachment of shear layer on the surface of the airfoil. Seshagiri et al. (2009) depicted that using of vortex generator suppresses the LSB and reduces the size of LSB. It is also observed that the vortex generator enhances the lift coefficient of NASA/Langley LS(1)- 0417 $\mathrm{GA}(\mathrm{W})-1$ airfoil up to $25 \%$ at $\mathrm{Re}=160000$. The effect of leading edge slot on the aerodynamic performance of NACA 4412 airfoil was explored by Beyhaghi and Amono (2018). They examined various slot configurations at different angles of attack and found that the maximum raise in lift to drag ratio is occurred at the angles of attack of $\alpha=$ $6^{\circ}$ and $8^{\circ}$. Weber et al. (2011) conducted a numerical study on flow over humpback whale flipper with and without leading edge perturbation. They found that the flipper with leading edge perturbation delays the flow separation significantly at higher angles of attack. Therefore it exhibits a higher lift coefficient in the poststall region in comparison with the flipper without leading perturbation. Zhang et al. (2013) modified the leading edge of NACA634-021 airfoil with sinusoidal wavy to enhance of airfoil aerodynamic at $\mathrm{Re}=50000$. They emphasized that the lift coefficient and the lift to drag ratio raises especially at poststall angles of attack, since generated streamwise vortices by the leading edge perturbation suppress the flow separation. Tong et al. (2018) numerically demonstrated that noise reduction could be obtained up to $9.5 \mathrm{~dB}$ by wavy leading edge. They indicated that reduction of wall pressure fluctuation and coherence length helps to suppress the noise. Faruqui et al. (2014) carried out numerical simulations to examine the effect of bumpy surface on the flow separation of NACA 4315 airfoil. They reported that flow separation is delayed up to angle of attack of $\alpha=15^{\circ}$ and also the lift coefficient significantly increases in comparison with the clean airfoil. Meena et al. (2018) performed a numerical investigation to explore the effect of gurney flap on the lift coefficient of various NACA symmetric profiles at $\mathrm{Re}=1000$. They noted that the gurney flap reduces the movement of flow from pressure side of airfoil to suction side of airfoil. Due to this reason, the lift to drag ratio increases up to twofold of baseline airfoil, however the drag coefficient increase especially at high angles of attack. Hafien et al. (2016) numerically demonstrated that using of flexible flaps on the suction side of NACA 0012 airfoil suppresses the vortex shedding and enhances the lift coefficient up to $69.49 \%$ at $\operatorname{Re}=342000$. Açıkel and Genç (2018) modified the suction surface of NACA 4412 airfoil with partially flexible membrane in order to control laminar separation bubble at $\mathrm{Re}=25000,50000$ and 75000. They emphasized that the flexible membrane is more effective in the suppression of laminar separation bubble at $\mathrm{Re}=25000$ and lift to drag ratio attains the twofold of the rigid airfoil. Zhang et al. (2018) expressed that the tripped Eppler E374 airfoil suppresses the laminar separation bubble, however, it leads to increase in the friction drag. They introduced that the drag reduction of the tripped airfoil could be achieved by using streamwise riblets at $\mathrm{Re}=200000$. Optimization of the cavity shape on Riso_B1_18 airfoil was studied by Fatehi et al. (2019) in order to enhance the aerodynamic performance of the wind turbine blade. They reported that formation of recirculation zone inside the cavity leads to delaying of flow separation. They also emphasized that lift to drag ratio of the airfoil could be raised up to $57 \%$ by optimized cavity in comparison with the without cavity profile. Genç et al. (2019) introduced using of roughness element to control flow around NACA 4412 airfoil. They demonstrated that the lift coefficient is increased approximately by $14 \%$ and stall angle is delayed from $14^{\circ}$ to $20^{\circ}$, since the increasing of momentum of flow in the suction side of airfoil by roughness element suppresses the LSB. Zuo et al. (2019) carried out numerical simulations to examine the influence of serrated trailing edge on flow characteristics of NACA 0018 at $\mathrm{Re}=160000$. They mentioned that tonal noise could be reduced due to the suppression of vortex shedding frequency by using serrated trailing edge.

On the other hand, trips have been applied as a passive control method. Huber and Mueller (1987) conducted an experimental study to investigate the influence of the trip wire on the aerodynamic performance of Wortmann FX 63-137 airfoil at Re $=10000$. They emphasized that the drag reduction and increasing of the lift to drag ratio could be achieved depending on the location and the thickness of the trip, since the trip is effective on the flow separation point, formation of LSB and transition of the boundary layer. Gopalarathnam et al. (2003) mentioned that the drag reduction of airfoil with trip is associated with decreasing of bubble drag. Because of this reason, the trip causes the drag reduction at low Reynolds number. Because decreasing of the size of LSB by trip reduces the bubble drag. However, it leads to 
increase in the drag coefficient at high Reynolds number, since the bubble drag is less effective at high Reynolds number. Traub (2011) studied experimentally flow over S8036 airfoil with trip strips and demonstrated that using of trip suppresses the laminar separation bubble and enhances the lift to drag ratio up to $46 \%$. Recently, Sreejith and Sathyabhama (2018) performed a numerical investigation to examine the effect of the trip on the formation of laminar separation bubble over E216 airfoil at $\operatorname{Re}=100000$. They showed that the laminar separation bubble could be eliminated by using trip. Moreover, the drag reduction is obtained up to $15.48 \%$ and the lift to drag ration is increased by $21.62 \%$.

Most of the studies about flow control with a rod or a trip in the literature focuses on the aerodynamic coefficients. However, exhibition of detailed mean and unsteady flow characteristics is essential in order to understand the flow control mechanism of airfoil with a control rod. In the present study, it was aimed to suppress the formation of LSB and the vortex shedding frequency by using a rod. In this sense, the present experimental investigation was performed to explore the effect of the diameter and location of the control rod at different angles of attack on the formation of LSB and the vortex shedding frequency of SD7062 airfoil using particle image velocimetry (PIV) at $\mathrm{Re}=30000$.

\section{EXPERIMENTAL SETUP}

An SD7062 airfoil model with control rod was tested in a water channel at Fluid Mechanics Laboratory of Cukurova University. The water channel consists of two reservoir and a test section. The test section of channel is $1 \times 0.75 \mathrm{~m}^{2}$ with a length of $8 \mathrm{~m}$. The inlet reservoir includes a settling chamber and a honeycomb in order to keep the turbulence intensity in the test section below $0.5 \%$. The water flow was driven by means of centrifugal pump. The airfoil model with $180 \mathrm{~mm}$ chord length, $\mathrm{c}$ and $400 \mathrm{~mm}$ span length, s was manufactured from Acrylonitrile Butadiene Styrene (ABS) material via $3 \mathrm{D}$ printer. In order to minimize the laser reflection, ABS material having black color was used. The control rods with three different diameters $(\mathrm{d}=3 \mathrm{~mm}, 6 \mathrm{~mm}$ and $8 \mathrm{~mm})$ were examined. The rod was located on the suction surface of the airfoil. The locations and diameters of rod were normalized with chord length and dimensionless parameters were defined as rod location $(\mathrm{x} / \mathrm{c}=0.4,0.5,0.6$ and 0.7$)$ and diameter ratio $(\mathrm{d} / \mathrm{c}=0.017,0.033$ and 0.044$)$. Also, the effect of rod on flow control was examined at angles of attack in the range of $0^{\circ}$ to $10^{\circ}$. Angle of attack from plan view was clockwise direction. A schematic representation of parameters is given in Fig.1. Uniform water flow speed was $U_{\infty}=165 \mathrm{~mm} / \mathrm{s}$ and corresponding Reynolds number based on the chord length was 30000 . The height of water was $550 \mathrm{~mm}$. Circular end plate was mounted on the upper side of the airfoil and the lower side of the airfoil was located bottom of the channel in order to ensure 2D flow.
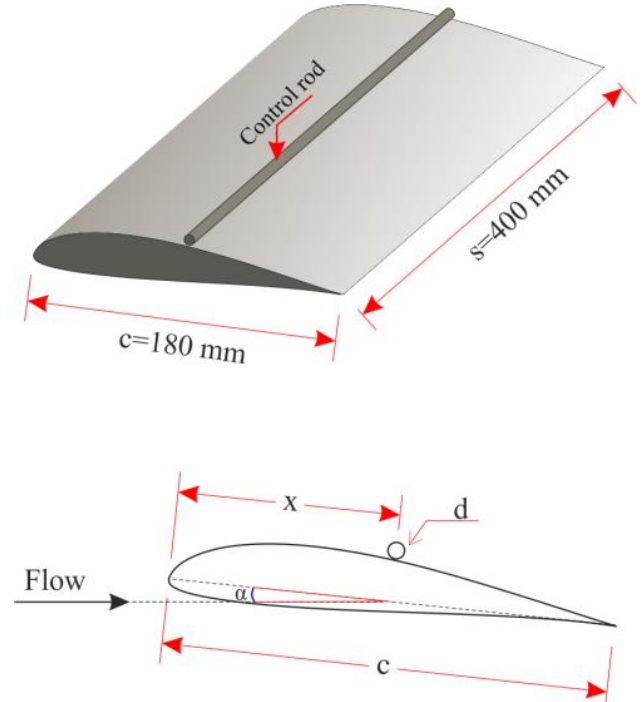

Fig. 1. A schematic representation of the airfoil, control rod.
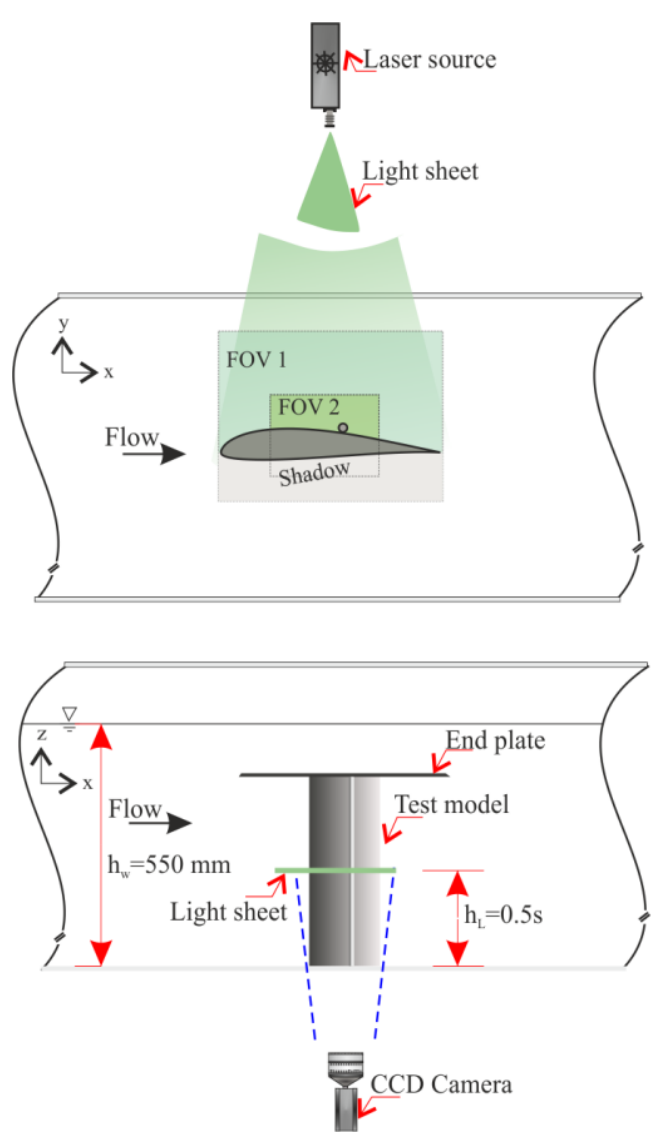

Fig. 2. A sketch of experimental set-up for plan view and side view.

Velocity measurements in the desired flow field were performed using Dantec Dynamic 2D particle image velocimetry system. Silver coated hollow glass spheres with $10 \mu \mathrm{m}$ diameter were used to seed the water flow. A double-pulsed Nd: Yag laser having a maximum power out of $120 \mathrm{~mJ}$ at $532 \mathrm{~nm}$ wavelength was used as light source to illuminate the field of view. Digital images were captured at 
an acquisition rate of $15 \mathrm{~Hz}$ by a CCD camera (Dantec Dynamics A/S, FlowSense 2M, 1600×1200 pixel). Light sheet in $2 \mathrm{~mm}$ thickness was located at the mid-span of the airfoil and the measurements were performed in the plan view. The time between pulses was set as $1.75 \mathrm{~ms}$ to obtain maximum amount of particle displacement. A sketch of experimental set-up is presented in Fig. 2. Measurements were carried out for two different fields of view designated as FOV I and FOV II in Fig. 2, in order to obtain both general and detailed information about the flow characteristics. FOV I covered an area of $1.15 \mathrm{c} \times 0.86 \mathrm{c}$ while FOV II covered an area of $0.6 \mathrm{c} \times 0.45 \mathrm{c}$. The number of image pair was set as 1000 for each test case and image processing was carried out using Dynamics Studio v4 software. The adaptive correlation technique including two step refinement with initial $128 \times 128$ pixels integration area and final $32 \times 32$ pixels integration area was applied with $50 \%$ overlap in order to obtain the raw velocity vectors. For each field of view, $99 \times 74$ velocity vectors were obtained. The local median filter technique were applied to detect erroneous vectors (less than $1 \%$ ) caused by reflection, shadow or laser sheet distortions and then these vectors were replaced by means of bilinear least-squares fit technique. Finally, the instantaneous and the time averaged flow characteristics were calculated. The uncertainty in the measurement plane was less than $2 \%$.

\section{RESULTS AND DISCUSSIONS}

PIV measurements initially were performed for the baseline airfoil at various angles of attack to reveal the general flow characteristics of the SD7062 airfoil. Figure 3 shows the flow structure of the baseline airfoil including time averaged streamline topologies superposed with normalized streamwise velocity contours and normalized Reynolds shear stress distributions at various angles of attack.

At $\alpha=0^{\circ}$, the time averaged streamline topology depicts that laminar separation bubble (LSB) which is designated as $\mathrm{LB}_{\mathrm{B}}$ occurs on the suction surface of the airfoil.

At the angle of attack of $\alpha=4^{\circ}$, it is observed formation of both the LSB and the trailing edge separation which is designated as $\mathrm{S}$ in Fig. 3. The zone having negative velocity values refers to existence of a recirculation region. It is found that the location of laminar separation bubble and the trailing edge separation move towards the leading edge of airfoil gradually up to $\alpha=6^{\circ}$ as increased angle of attack. On the other hand, a different flow behavior is observed at $\alpha=8^{\circ}$. The separated boundary layer reattaches the trailing edge of the airfoil and a larger recirculation zone having two foci (F1 and F2) occurs over the airfoil surface, since the laminar separation bubble interacts with the trailing edge separation and leads to occurrence of bubble bursting. Therefore, the boundary layer fails to reattach to the surface of airfoil and an abrupt stall occurs. Similar flow behavior is also observed at $\alpha=10^{\circ}$.

On the other hand, the flow topology obtained from the PIV measurements indicates that stall occurs at $\alpha=12^{\circ}$. The Reynolds shear stress distributions demonstrate that the value of maximum Reynolds shear stress raises with the increasing angle of attack. However, the location of maximum Reynolds shear stress move towards the leading edge up to $\alpha=6^{\circ}$ with the increasing angle of attack. On the other hand, it move towards the trailing edge for $\alpha \geq 8^{\circ}$.

Moreover, the location of Reynolds shear stress having 0.001 value which is designated in Fig. 3 refers to start transition of turbulence boundary layer (Lian and Shyy 2007) It is observed that the location of transition point gets close the leading edge gradually as the angle of attack increased. Detailed information about the formations of laminar separation bubble, transition and bubble bursting was reported by Tani (1964); O'Meara and Mueller (1987); Yarusevych et al. (2009).

In order to obtain detailed information about the flow characteristics of the baseline airfoil, Fig.4 shows obtained results from FOV II at $\alpha=6^{\circ}, 8^{\circ}$ and $10^{\circ}$. At $\alpha=6^{\circ}$, the time averaged streamline topology depicts that the laminar separation bubble covers approximately $33 \%$ of suction surface of the airfoil. The maximum turbulent kinetic energy and maximum $u_{r m s}$ occur near the reattachment of the boundary layer to the airfoil surface. It is observed that the strong interaction occurs between the LSB and the trailing edge separation at $\alpha=8^{\circ}$. Therefore, the values of maximum TKE and maximum $\mathrm{u}_{\mathrm{rms}}$ are higher than that obtained from $\alpha=6^{\circ}$. On the other hand, the interaction of LSB with the trailing edge separation decreases at $\alpha=10^{\circ}$, since LSB merges with the trailing edge separation and behaves like a single foci. Therefore, the values of maximum TKE and maximum $\mathrm{u}_{\mathrm{rms}}$ decrease in comparison with the angle of attack of $\alpha=8^{\circ}$.

The effect of rod on flow characteristics of the airfoil was examined at three angles of attack which are $\alpha=6^{\circ}, 8^{\circ}$ and $10^{\circ}$. Figure 5 depicts the time averaged streamline topology superposed with the normalized streamwise velocity for diameter ratio of $d / c=0.033$ at different chordwise locations. It is seen that the location of rod plays an important role on the flow structure over the airfoil. When the rod is located at $\mathrm{x} / \mathrm{c}=0.4$, it causes earlier stall for all examined angles of attack, since the separated flow fails to reattach to the surface of the airfoil. Moreover, the strong recirculation region occurs on the suction surface of the airfoil. At $\mathrm{x} / \mathrm{c}=0.5$, the rod worsens the flow structure over the airfoil for $\alpha$ $=6^{\circ}$, since it leads to the occurrence of large recirculation region (laminar separation bubble) over the airfoil surface. LSB and the trailing edge separation kept own identity. However, the rod causes reduction in the height of recirculation zone and also the formation of single focus at $\alpha=8^{\circ}$ and $10^{\circ}$. At $\mathrm{x} / \mathrm{c}=0.6$, the rod remarkably suppresses the formation of LSB for $\alpha=6^{\circ}$. Moreover, it significantly reduces the recirculation zone in the 

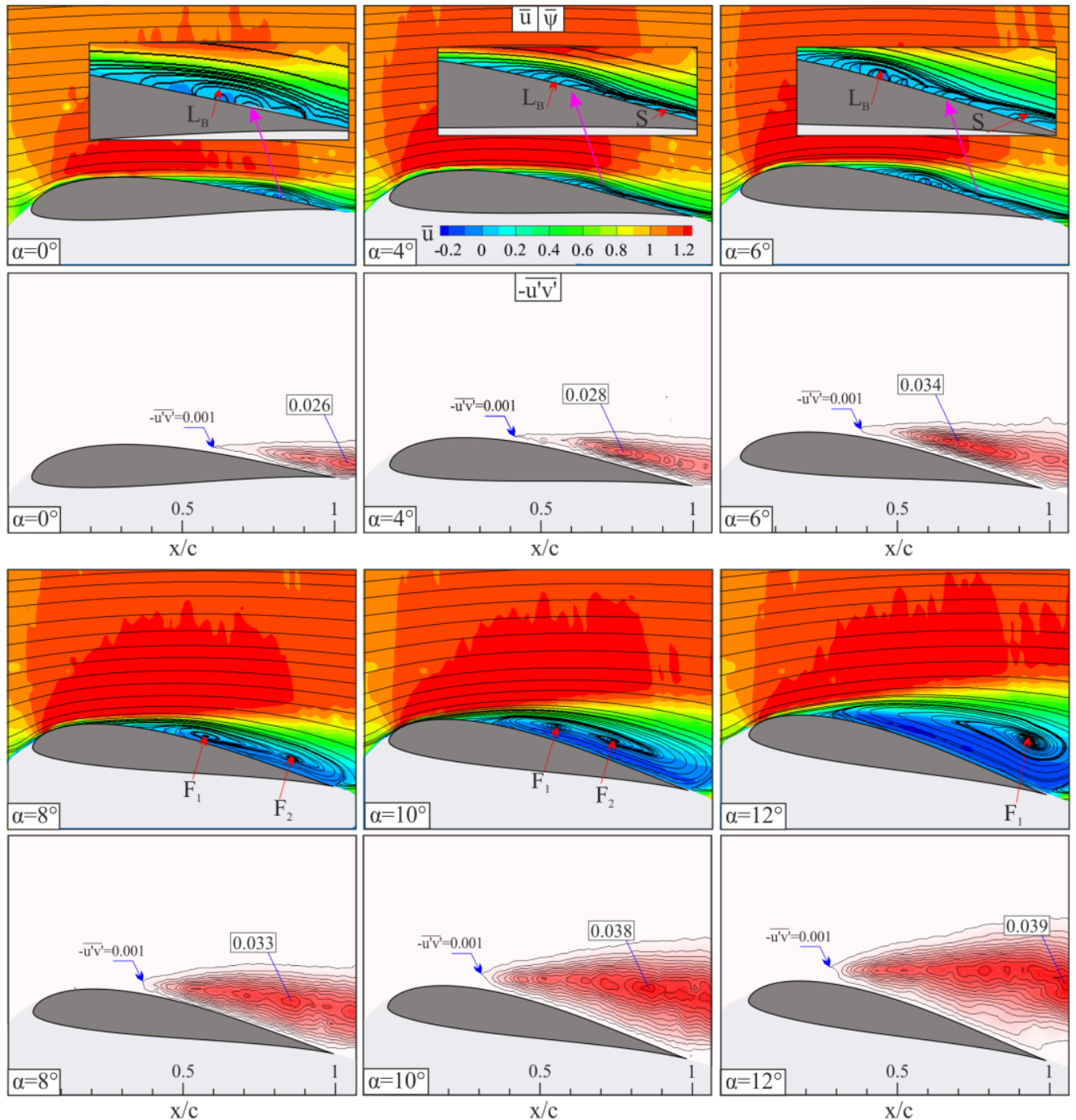

Fig. 3. Time averaged flow characteristics over the baseline airfoil at different angles of attack.

size for $\alpha=8^{\circ}$ while the rod divides the recirculation zone into two regions and leads to occurrence of two foci. At $\mathrm{x} / \mathrm{c}=0.7$, the rod worsens the flow structure, especially for $\alpha=8^{\circ}$ and $10^{\circ}$ when compared to the results that obtained from the rod location of $\mathrm{x} / \mathrm{c}=0.6$. Two-well defined foci formation are observed for $\alpha=8^{\circ}$ and the height of the recirculation zone increases in comparison with the rod located at $x / c=0.6$. In the light of obtained results from the PIV measurement, it can be concluded that flow control could be achieved depending on the location of the rod. The most effective locations are determined as $\mathrm{x} / \mathrm{c}=0.5$ and 0.6 . The effect of the diameter ratio of the rod on the flow structure over the airfoil will be discussed in the next section for these indicated control rod locations.

Figure 6 illustrates the influence of the diameter ratio on the flow characteristics over the airfoil for various angles of attack when the rod located at $\mathrm{x} / \mathrm{c}$ $=0.5$. It is found that the height of LSB is significantly reduced and the maximum $\mathrm{u}_{\mathrm{rms}}$ slightly decreases for $\mathrm{d} / \mathrm{c}=0.017$ at $\alpha=6^{\circ}$ compared to the baseline airfoil. However, it is observed that the increasing diameter ratio extends the size of recirculation zone and leads to occurrence of stall at $\alpha=6^{\circ}$. At $\alpha=8^{\circ}$, flow behavior is significantly altered by the diameter ratio of $\mathrm{d} / \mathrm{c}=0.017$. The separated boundary layer reattaches to surface of the airfoil at approximately $\mathrm{x} / \mathrm{c}=0.75$ while the boundary layer fails to reattaching the suction surface of the airfoil for $\mathrm{d} / \mathrm{c}=0.033$ and 0.044 . However, maximum $\mathrm{u}_{\mathrm{rms}}$ decreases with the increasing diameter ratio, since the rod suppresses the interaction of LSB with the trailing edge separation. At $\alpha=10^{\circ}$, the diameter ratio of $\mathrm{d} / \mathrm{c}=$ 0.017 leads to occurrence of two distinct foci that are far apart the each other. The interaction of these foci causes the increasing of maximum $u_{r m s}$ when compared to the baseline airfoil case. On the other hand, this interaction gradually diminishes with the increasing diameter ratio. Eventually, maximum $\mathrm{u}_{\mathrm{rms}}$ decreases up to 0.3 for $\mathrm{d} / \mathrm{c}=0.044$ at $\alpha=10^{\circ}$. 


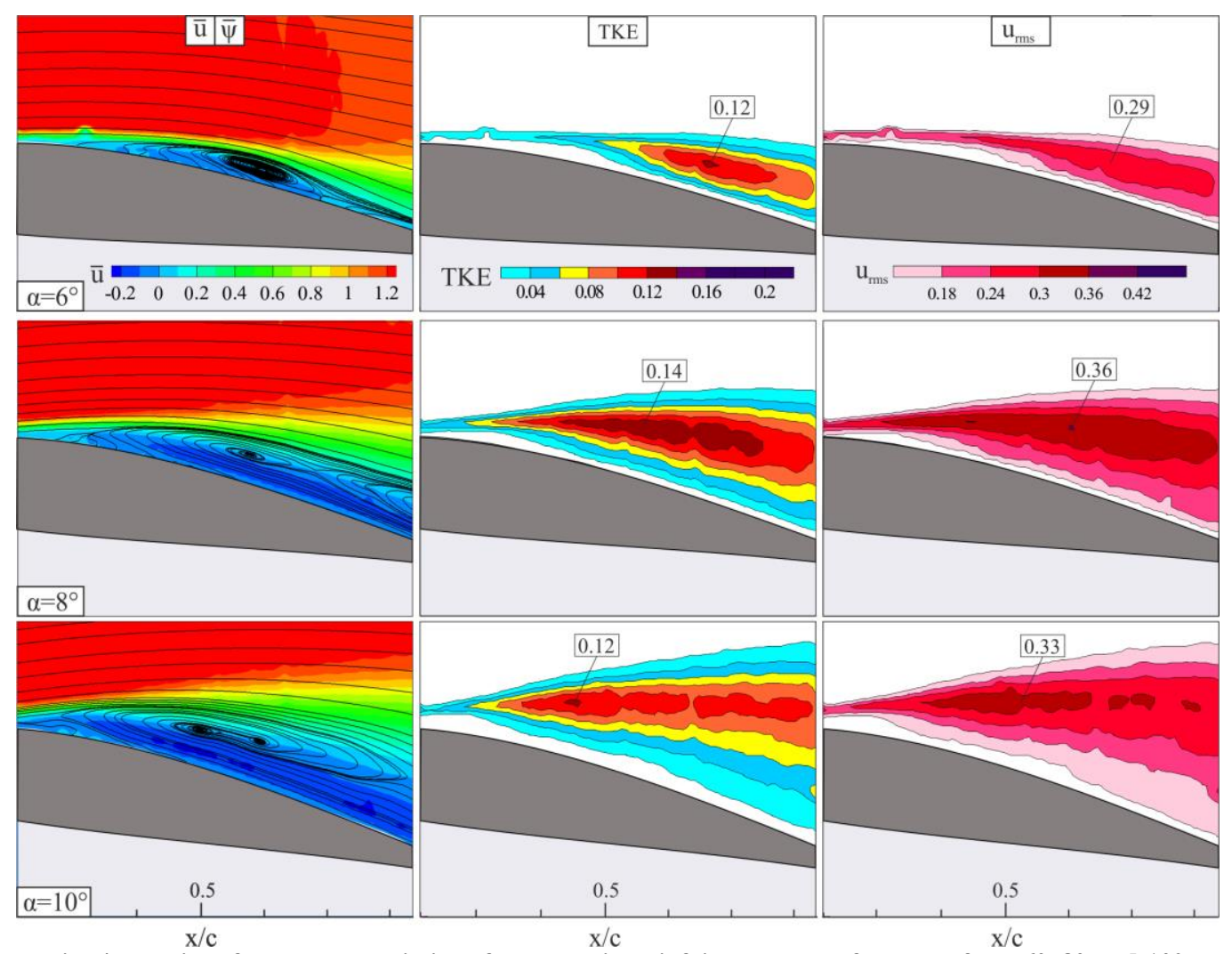

Fig. 4. Detailed flow characteristics of the baseline airfoil at angles of attack of $\alpha=6^{\circ}, 8^{\circ}$ and $10^{\circ}$.

The effect of the diameter ratio of the rod located at $\mathrm{x} / \mathrm{c}=0.6$ on flow characteristics over the suction surface of the airfoil is depicted in Fig. 7 for different angles of attack. It is found that the recirculation zone is divided into two regions as observed when the rod installed at $\mathrm{x} / \mathrm{c}=0.5$. However, the size of region occurred in front of the rod is larger than that the rod located at $\mathrm{x} / \mathrm{c}=0.5$. At $\alpha=8^{\circ}$, maximum urms is reduced by $22 \%$ for $\mathrm{d} / \mathrm{c}$ $=0.033$ and 0.044 in comparison with the baseline airfoil. The effect of the diameter ratio on the maximum $\mathrm{u}_{\mathrm{rms}}$ is interesting at $\alpha=10^{\circ}$. When compared with the baseline airfoil, maximum $\mathrm{u}_{\mathrm{rms}}$ is increased by $6 \%$ for $\mathrm{d} / \mathrm{c}=0.017$ while it is nearly same for $\mathrm{d} / \mathrm{c}=0.033$ and 0.044 .

To show the effect of the rod installed at $\mathrm{x} / \mathrm{c}=0.5$ on the boundary layer, Fig. 8 presents normalized streamwise velocity profiles at various chordwise sections for different cases. The origin of $y / c$ is designated as the suction surface of the airfoil. At $\alpha$ $=6^{\circ}$, diameter ratio of $\mathrm{d} / \mathrm{c}=0.017$ leads to increasing of momentum in the boundary layer. Therefore, the height of the boundary layer is reduced up to $22 \%$, especially at $\mathrm{x} / \mathrm{c}=0.5$ and 0.6 in comparison with the baseline airfoil. However, the rod blocked the further flow with the increasing diameter ratio and causes formation of separated region in both the rear and the front of the rod. The formation of large scale separated region in the rear of the rod prevents attaching of the boundary layer to surface of the airfoil. Therefore, the height of boundary layer increases particularly at $\mathrm{x} / \mathrm{c}=0.6$ and 0.7 in comparison with the baseline airfoil. The negative velocity profile at $\mathrm{x} / \mathrm{c}=0.6$ and 0.7 indicates that the recirculation region is dominant on the flow structure over the airfoil. At $\alpha=8^{\circ}$, the most effective reduction in the height of the boundary layer (approximately 22\%) is obtained at $\mathrm{x} / \mathrm{c}=0.6$ for $\mathrm{d} / \mathrm{c}=0.017$. It is observed that $\mathrm{d} / \mathrm{c}=$ 0.017 reduces not only the height of boundary layer but also the recirculation region size in the horizontal plane. It is found that diameter ratios of $\mathrm{d} / \mathrm{c}=0.033$ and 0.044 have insignificant effect on the height of the boundary layer. On the other hand, the height of the boundary layer is lower than the baseline airfoil for all diameter ratios at $\alpha=10^{\circ}$. The height of the boundary layer decreases with the increasing diameter ratio. Because the height of the boundary layer raises with the increasing angle of attack and large scale recirculation region covers the suction surface of the airfoil. Therefore, the effect of the control cylinder having low diameter ratio on the momentum of the boundary layer is lower. On the other hand, the increasing dimeter ratio enhances the momentum transfer of boundary layer especially above the rod and gets dominate on the formation of the recirculation region in the rear of the rod. The height of boundary layer is reduced by $17 \%$ at $\mathrm{x} / \mathrm{c}=0.5$ for $\mathrm{d} / \mathrm{c}=0.044$ when compared to the baseline airfoil. However, it is obtained that negative velocity profiles are nearly same for all cases at $\alpha=10^{\circ}$. Consequently, it is clearly seen that the rod is affective on the reducing of the height of the boundary layer depending on the 

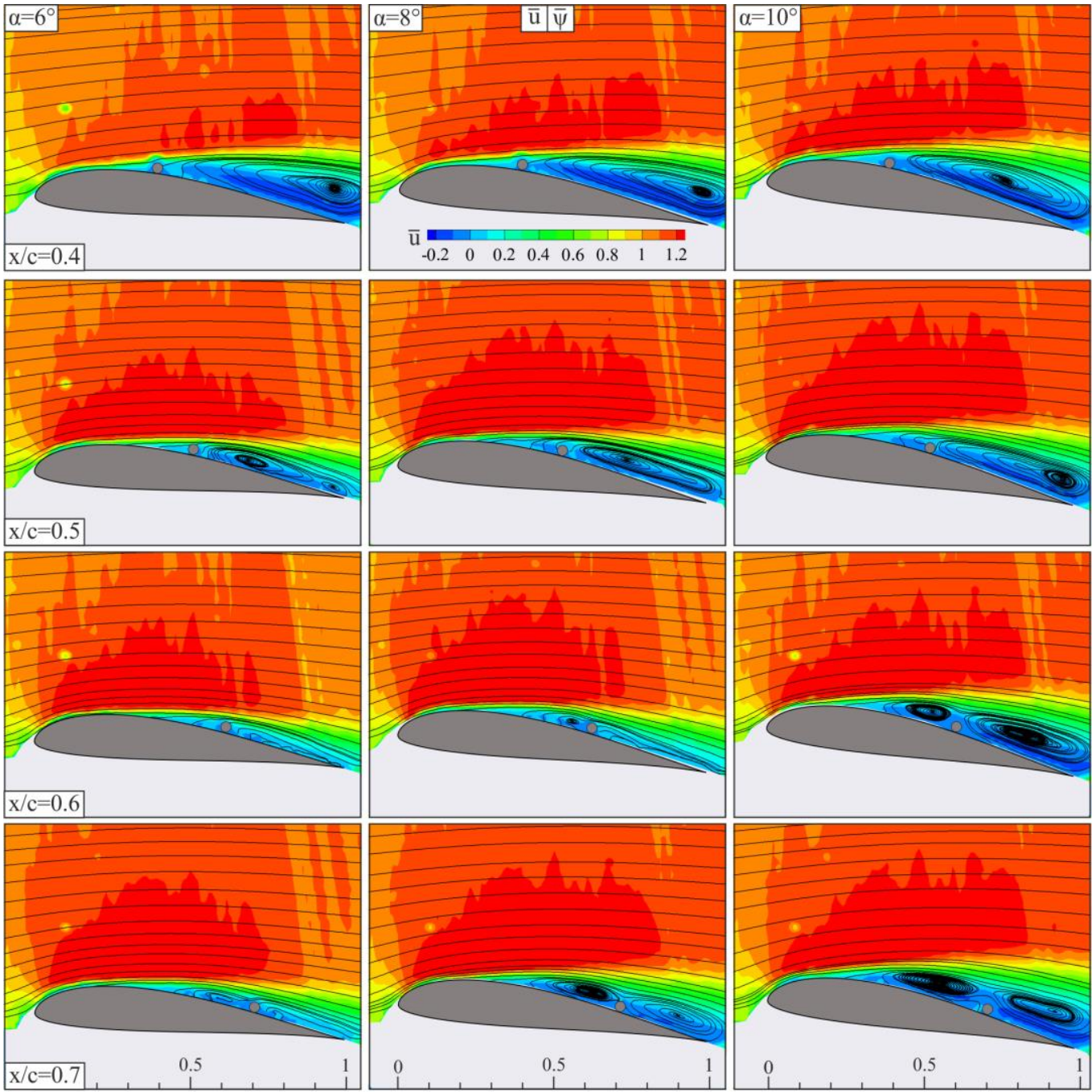

$\mathrm{x} / \mathrm{c}$

$\begin{array}{ccc}\mathrm{x} / \mathrm{c} & \mathrm{x} / \mathrm{c} & \mathrm{X} / \mathrm{c} \\ \text { Fig. 5. Distribution of streamline topology superposed with the mean streamwise velocity over the }\end{array}$

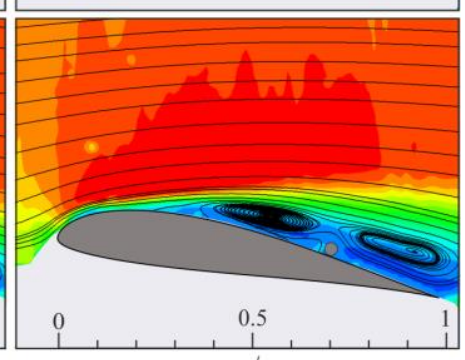
airfoil for $\mathrm{d} / \mathrm{c}=\mathbf{0 . 0 3 3}$ at various angles of attack and rod locations.

diameter ratio, especially at $\mathrm{x} / \mathrm{c}=0.5$ and 0.6 for all tested angles of attack. Moreover, the decreasing of the height of the boundary layer by the rod could be evaluated as an indicator of reduction of the drag coefficient. Yayun et al. (2015) emphasized that the height of the boundary layer affects directly pressure coefficient distribution thereby aerodynamic coefficients. They noted that thinner boundary layer leads to reduction of the drag coefficient.

The normalized streamwise velocity profiles when the rod installed at $\mathrm{x} / \mathrm{c}=0.6$ are presented in Fig. 9 for various cases. It is observed that the effect of rod at $\alpha=6^{\circ}$ on the height of the boundary layer is nearly similar with the rod located at $\mathrm{x} / \mathrm{c}=0.5$ for $\mathrm{d} / \mathrm{c}=0.017$. However, the diameter ratio of $\mathrm{d} / \mathrm{c}=$ 0.033 leads to an increase, which is approximately by $28 \%$, in the height of boundary layer, since the rod causes formation of large scale recirculation region over the airfoil compared to the baseline airfoil. Due to this reason, PIV measurement was not performed for diameter ratio of $\mathrm{d} / \mathrm{c}=0.044$ at $\alpha$ $=6^{\circ}$. It is observed that the height of the boundary layer decreases at $\mathrm{x} / \mathrm{c} \geq 0.5$ for $\alpha=8^{\circ}$ and $10^{\circ}$ compared to the baseline airfoil. However, this reduction is lower than the case which the rod located at $\mathrm{x} / \mathrm{c}=0.5$ since the strong recirculation region occurs in front of the rod. The most effective diameter ratios at $\alpha=8^{\circ}$ and $10^{\circ}$ are obtained as $\mathrm{d} / \mathrm{c}$ $=0.017$ and 0.044 , respectively. It is observed that the height of boundary layer reduces up to $13 \%$ at $\alpha$ $=8^{\circ}$ while it diminishes up to $17 \%$ at $\alpha=10^{\circ}$.

In order to reveal the mechanism of rolling up of the shear layers, Fig. 10 illustrates instantaneous normalized vorticity contours at different time steps for the baseline airfoil and most effective diameter ratios on reduction of the height of the boundary layer. As observed from PIV measurement results for the baseline airfoil at $\alpha=6^{\circ}$, the separated shear layer rolls up due to the Kelvin-Helmholtz instabilities, then the separated boundary layer reattaches to the surface of the airfoil and causes the formation of the LSB. On the other hand, instantaneous vorticity distributions of the baseline 
T. Durhasan / JAFM, Vol. 13, No. 6, pp. 1819-1833, 2020.

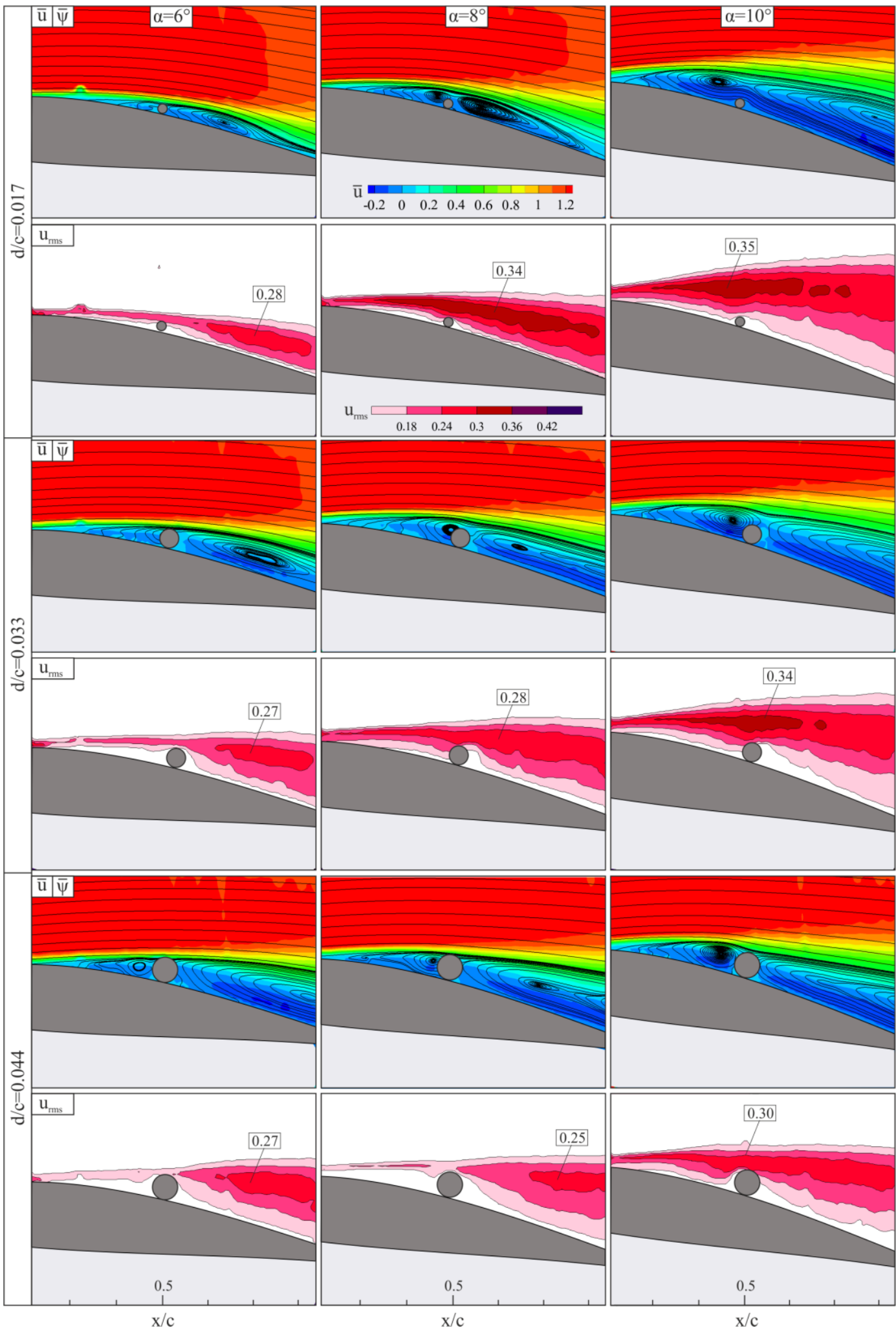

Fig. 6. Flow characteristics over airfoil when the rod located at $\mathrm{x} / \mathrm{c}=\mathbf{0 . 5}$ for $\mathrm{different} \mathrm{d} / \mathrm{c}$ and angles of attack.

airfoil at $\alpha=8^{\circ}$ and $10^{\circ}$ depict occurrence of stall. The separated boundary layer does not reattach to the surface of the airfoil and onset of rolling up of the shear layer closes to the leading edge with the increasing angle of attack. Also, the shed vortices interact with the positive sign vortices stemmed from the trailing edge separation and ends up with the eruption of positive sign vortices. 


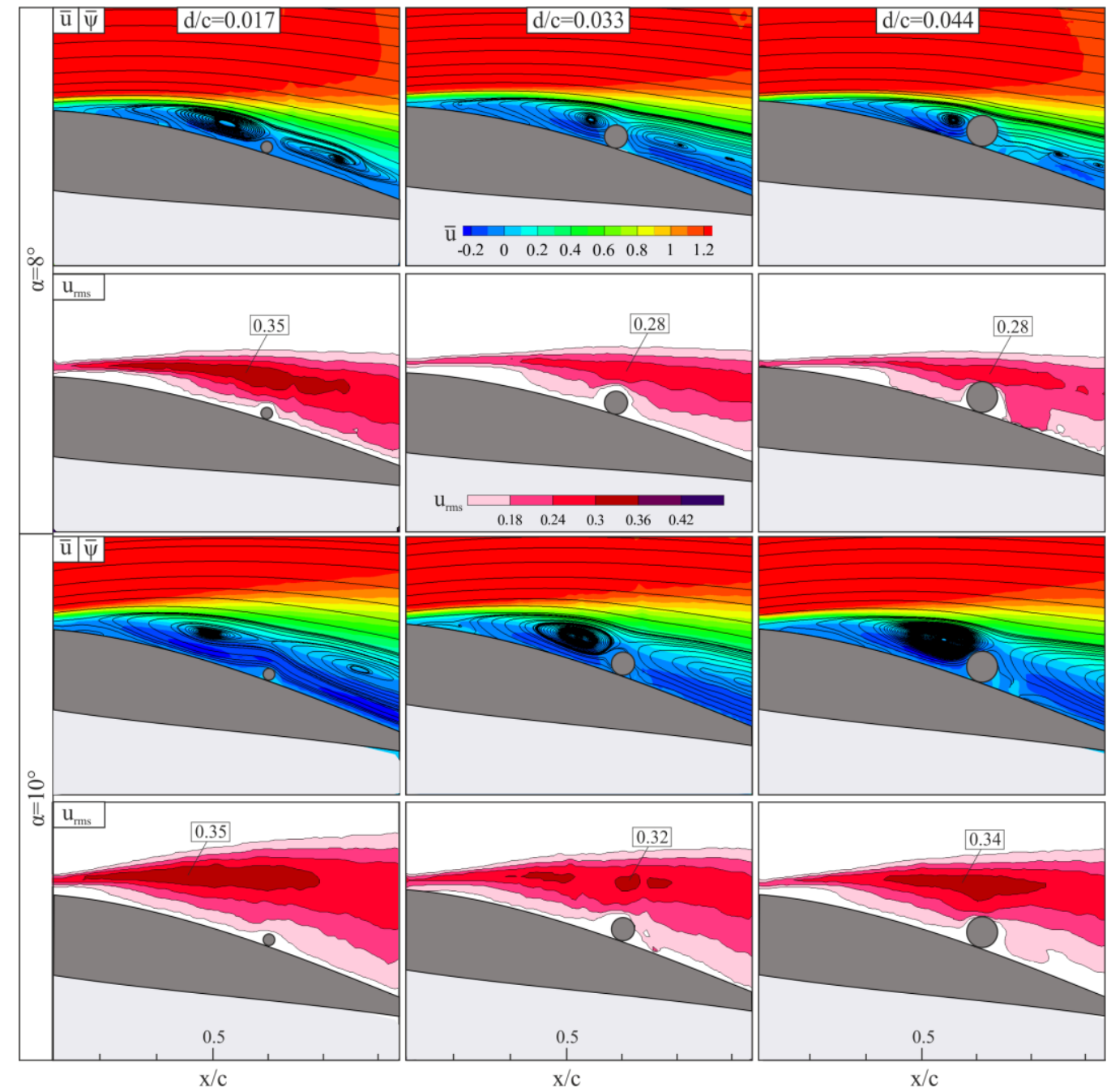

Fig. 7. Flow characteristics over the airfoil when the rod located at $x / c=0.6$ for $\operatorname{different} d / c$ and angles of attack.

Therefore, the shedding of vortices become more unsteady over the suction surface of the airfoil. On the other hand, using of the rod restricts movement of secondary vortices (positive sign vortices) towards the leading edge. For this reason, secondary vortices could not grow as much as the baseline airfoil. Moreover, the shear layer elongates further chordwise location of the airfoil and onset of rolling up of vortices moves toward the trailing edge in comparison with the baseline airfoil. Therefore, the height of boundary layer is reduced and unsteady vortices over suction surface of airfoil are suppressed by the rod. It should be noted that elongated shear layer interacts with the rod at $\alpha=8^{\circ}$ and $10^{\circ}$ when the rod located at $\mathrm{x} / \mathrm{c}=0.5$.

Power spectral density distributions of the streamwise velocity are presented in Fig. 11 for the baseline airfoil and the most effective cases that reducing the height of the boundary layer. PSD results are presented for two locations which are designated as P1 and P2. P1 is selected near the shedding of shear layer to determine the frequency of vortex shedding while P2 is selected at further chordwise location in order to show nonlinear interaction of vortices. Corresponding Strouhal number of the dominate frequency of vortex shedding is determined as 0.23 at P1 for $\alpha=6^{\circ}$ and St value gradually increases with the increasing angle of attack, since shedding of vortices decreases in the size. St values significantly increase at P2 for all angles of attack due to increasing of unsteadies stemmed from the interaction of vortices as mentioned in Fig. 9. Ebrahimi and Hajipour (2018) emphasized that dominant frequency is obtained different at the shear layer and trailing edge due to the changing of the separated flow mode.

On the other hand, St value of P1 is same with the baseline airfoil when using the rod, however, it decreases by $34 \%$ for P2 at $\alpha=6^{\circ}$, since the interaction of vortices is suppressed. At $\alpha=8^{\circ}$ and $10^{\circ}$, using of the rod reduces the dominant frequency at not only P1 but also P2, since the rod leads to delaying of rolling up of the shear layer and also suppresses the interaction of vortices in comparison with the base line airfoil. Also, they have same St value at both $\alpha=8^{\circ}$ and $\alpha=10^{\circ}$. One possible reason of this situation, the shear layer elongates towards the trailing edge and interacts 


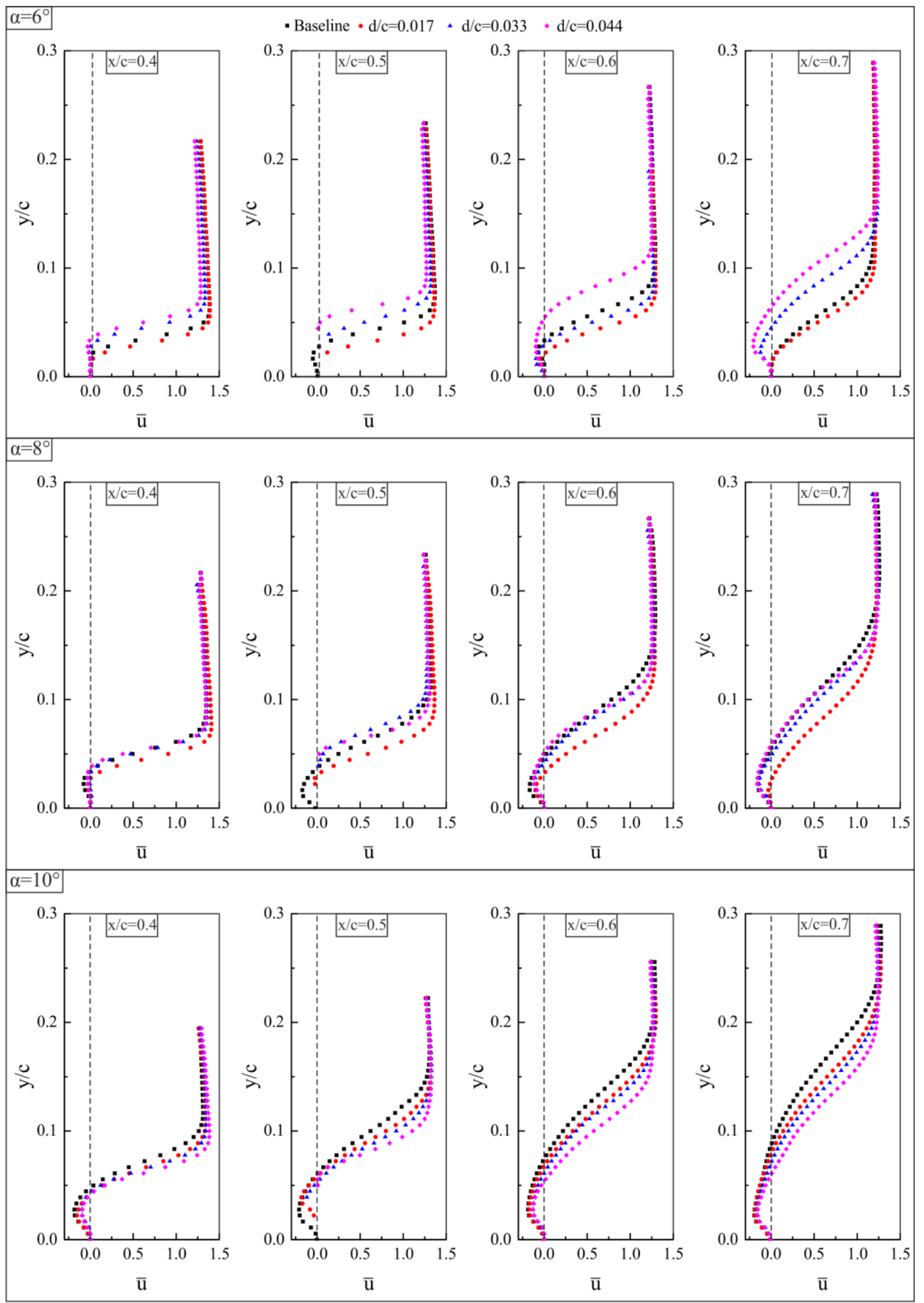

Fig. 8. Mean streamwise velocity profiles over the airfoil when the rod installed at $x / c=0.5$ at different chordwise locations and angles of attack for $\mathrm{d} / \mathrm{c}=0.017,0.033$ and 0.044 .

with the rod at $x / c=0.5$. Therefore, the shear layer is split into small scale vortices by the rod. When compared to the baseline airfoil, $\mathrm{St}$ value is decreased by $\% 24$ and $\% 41$ at $\mathrm{P} 1$ and $\mathrm{P} 2$, respectively for $\alpha=10^{\circ}$.

\section{CONCLUSION}

An experimental study was performed using Particle image velocimetry technique (PIV) to reveal the influence of the rod on flow control over 


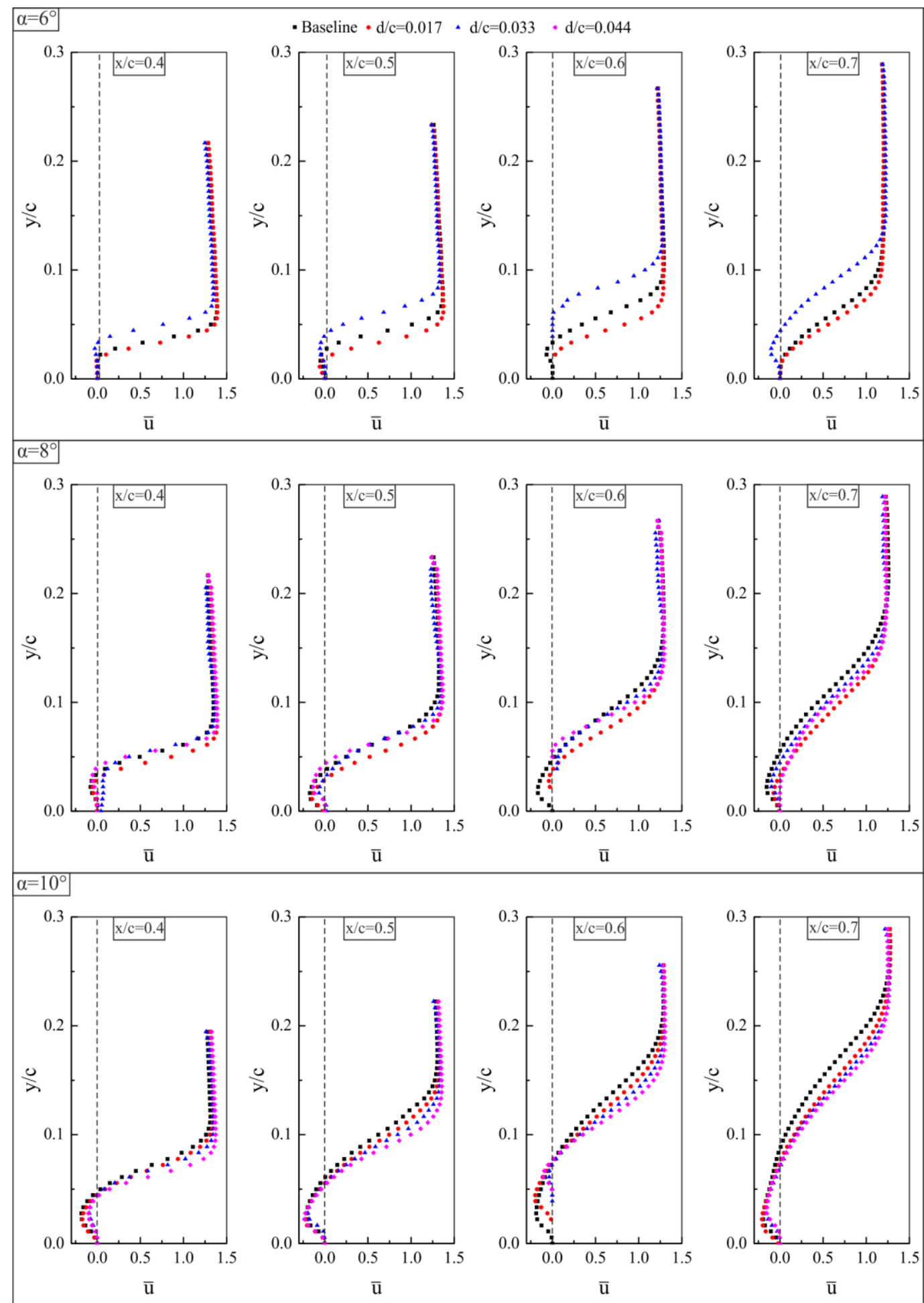

Fig. 9. Mean streamwise velocity profiles over the airfoil when the rod installed at $\mathrm{x} / \mathrm{c}=0.6$ at different chordwise locations and angles of attack for $\mathrm{d} / \mathrm{c}=\mathbf{0 . 0 1 7}, \mathbf{0 . 0 3 3}$ and 0.044 .

SD 7062 airfoil at Reynolds number of $\operatorname{Re}=30000$. The main parameters are rod location, diameter ratio and angle of attack. The results depict that the flow characteristics are significantly affected by these parameters. The most effective control is achieved when the rod located at $\mathrm{x} / \mathrm{c}=0.5$.
However, the effective diameter ratio depends on the angle of attack. It is observed that the diameter ratio of $\mathrm{d} / \mathrm{c}=0.017$ is the most effective to control flow at $\alpha=6^{\circ}$ and $8^{\circ}$ whereas the diameter ratio of $\mathrm{d} / \mathrm{c}=0.044$ is the most effective at $\alpha=10^{\circ}$. The height of the boundary layer is reduced up to $22 \%$ 
T. Durhasan / JAFM, Vol. 13, No. 6, pp. 1819-1833, 2020.

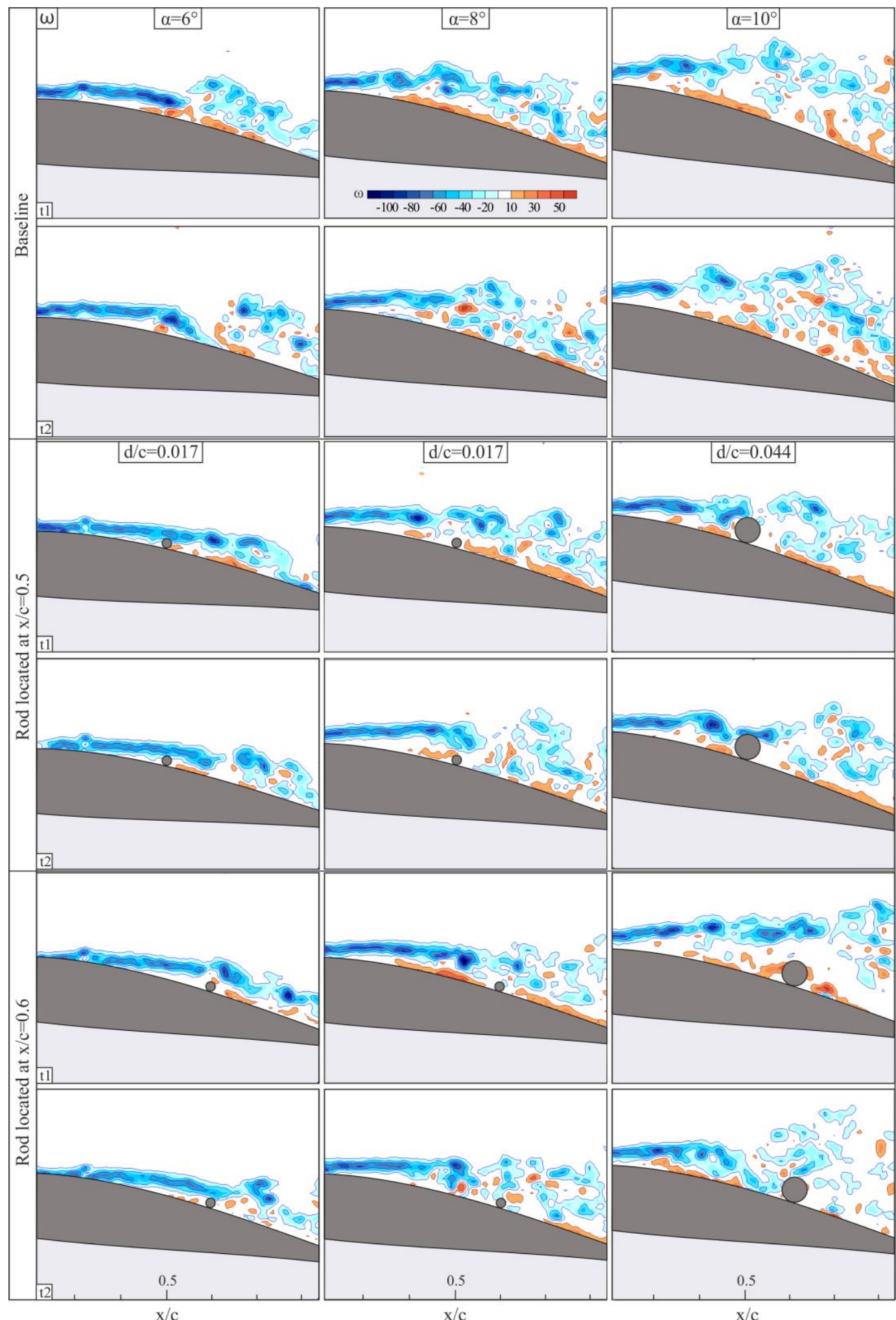

Fig. 10. Instantaneous vorticity disruptions over the airfoil with and without rods at different time steps.

at angles of attack $\alpha=6^{\circ}$ and $8^{\circ}$ for the diameter ratio of $\mathrm{d} / \mathrm{c}=0.017$ in comparison with the baseline airfoil while it diminishes up to $17 \%$ at $\alpha=10^{\circ}$ for $\mathrm{d} / \mathrm{c}=0.044$. The rod restricts the movement of secondary vortices towards the leading edge of the airfoil. Therefore, secondary vortices could not grow as much as the baseline airfoil. Thus, unsteady vortices over the suction surface of the airfoil are suppressed by the rod. Power spectra density analysis demonstrated that the dominant frequency 


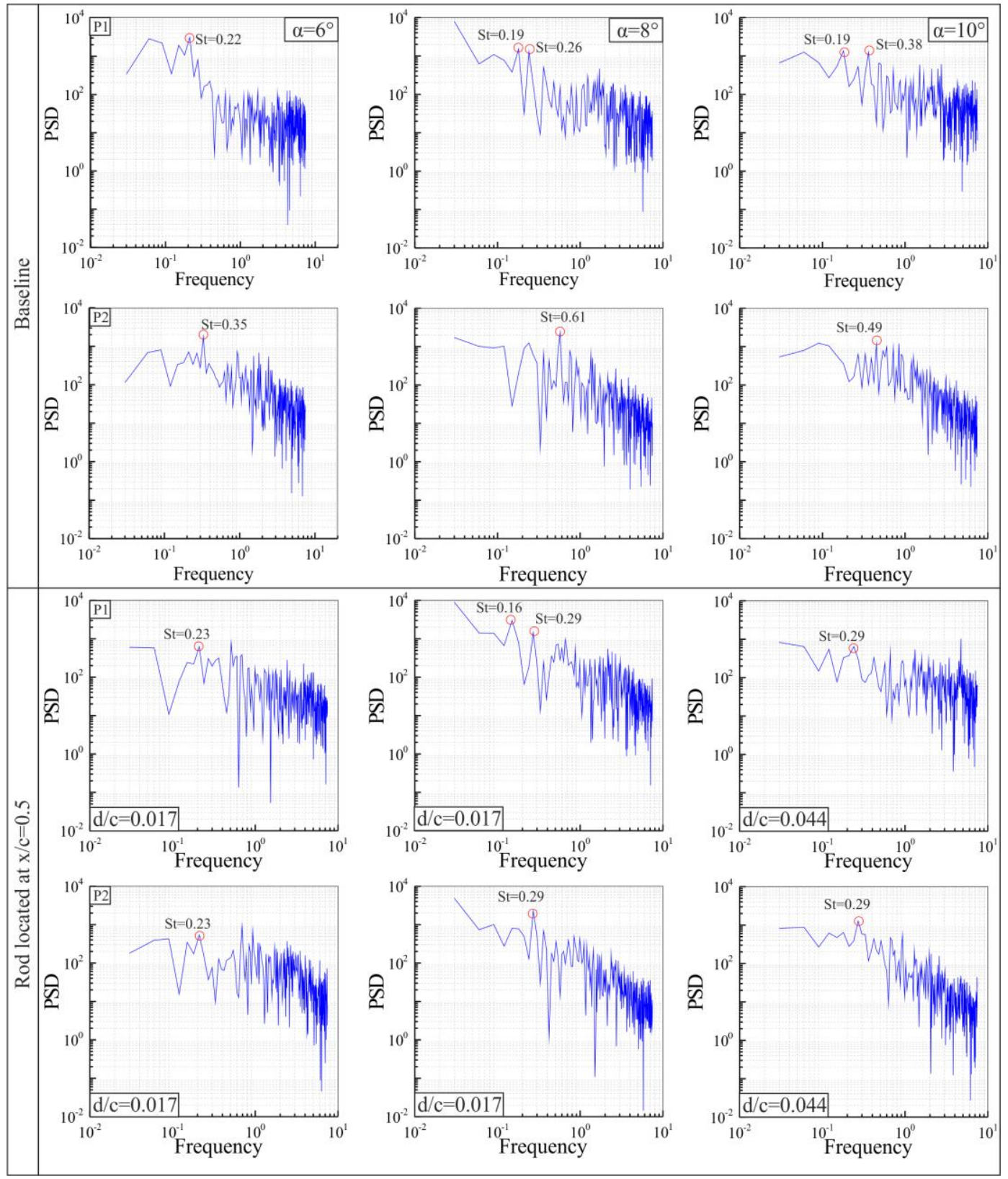

Fig. 11. PSD analysis of streamwise velocity over the airfoil with and without rods.

is reduced up to $34 \%$. It is noted that flow control of airfoil could be achieved by means of proper the rod location and diameter ratio, otherwise the rod affects adversely the flow characteristics over the airfoil.

\section{ACKNOWLEDGEMENTS}

The author would like to thank the Department of Mechanical Engineering at University of Cukurova to supply the Fluid Mechanics Laboratory.

\section{REFERENCES}

Açıkel, H. H. and M. S. Genç (2018). Control of laminar separation bubble over wind turbine airfoil using partial flexibility on suction surface. Energy 165, 176-190.

Akbiyık, H., H. Yavuz and Y. E. Akansu (2017). Comparison of the linear and spanwisesegmented DBD plasma actuators on flow control around a NACA0015 airfoil. IEEE Transactions on Plasma Science 45(11), 29132921.

Beyhaghi, S. and R. S. Amano (2018). A parametric study on leading-edge slots used on wind turbine airfoils at various angles of attack. Journal of Wind Engineering and Industrial Aerodynamics 175, 43-52. 
Burgmann, S., C. Brücker and W. Schröder (2006). Scanning PIV measurements of a laminar separation bubble. Experiments in Fluids 41(2), 319-326.

Ebrahimi, A. and M. Hajipour (2018). Flow separation control over an airfoil using dual excitation of DBD plasma actuators. Aerospace Science and Technology 79, 658668.

Faruqui, S. H. A., M. A. Al Bari, M. Emran and A. Ferdaus (2014). Numerical analysis of role of bumpy surface to control the flow separation of an airfoil. Procedia Engineering 90, 255260.

Fatehi, M., M. Nili-Ahmadabadi, O. Nematollahi, A. Minaiean and K. C. Kim (2019). Aerodynamic performance improvement of wind turbine blade by cavity shape optimization. Renewable Energy 132, 773-785.

Genç, M. S., H. H. Açıkel, M. T. Akpolat, G. Özkan and İ. Karasu (2016). Acoustic control of flow over NACA 2415 airfoil at low Reynolds numbers. Journal of Aerospace Engineering 29(6), 04016045.

Genç, M. S., K. Kemal and H. H. Açıkel (2019). Investigation of pre-stall flow control on wind turbine blade airfoil using roughness element. Energy 176, 320-334.

Gopalarathnam, A., B. A. Broughton, B. D. McGranahan and M. S. Selig (2003). Design of low Reynolds number airfoils with trips. Journal of Aircraft 40(4), 768-775.

Hafien, C., A. Bourehla and M. Bouzaien (2016). Passive Separation Control on a Symmetric Airfoil via Elastic-Layer. Journal of Applied Fluid Mechanics 9(5), 2596-2580.

Huang, L., P. G. Huang, R. P. LeBeau and T. Hauser (2004). Numerical study of blowing and suction control mechanism on NACA0012 airfoil. Journal of Aircraft 41(5), 1005-1013.

Huber, A. F. and T. J. Mueller (1987). The effect of trip wire roughness on the performance of the Wortmann FX 63-137 airfoil at low Reynolds numbers. Experiments in Fluids 5(4), 263-272.

Khoshkhoo, R. and A. Jahangirian (2016). Numerical simulation of flow separation control using multiple DBD plasma actuators. Journal of Applied Fluid Mechanics 9(4), 1865-1875.

Lee, S. J. and Y. G. Jang (2005). Control of flow around a NACA 0012 airfoil with a microriblet film. Journal of Fluids and Structures 20(5), 659-672.

Lian, Y. and W. Shyy (2007). Laminar-turbulent transition of a low Reynolds number rigid or flexible airfoil. AIAA Journal 45(7), 15011513.

Meena, M. G., K. Taira and K. Asai (2017). Airfoil- wake modification with gurney flap at low reynolds number. AIAA Journal 56(4), 13481359.

Meena, M. G., K. Taira and K. Asai (2018). Airfoilwake modification with gurney flap at low Reynolds number. AIAA Journal 56(4), 13481359.

O'Meara, M. M. and T. J. Mueller (1987). Laminar separation bubble characteristics on an airfoil at low Reynolds numbers. AIAA Journal 25(8), 1033-1041

Ricci, R., S. Montelpare and E. Silvi (2007). Study of acoustic disturbances effect on laminar separation bubble by IR thermography. Experimental Thermal and Fluid Science 31(4), 349-359.

Rinoie, K. and N. Takemura (2004). Oscillating behaviour of laminar separation bubble formed on an aerofoil near stall. The Aeronautical Journal 108(1081), 153-163.

Rinoie, K., M. Okuno and Y. Sunada (2009). Airfoil stall suppression by use of a bubble burst control plate. AIAA Journal 47(2), 322330 .

Sefiddashti, M. N., M. Nili-Ahmadabadi and B. S. Rizi (2018). Experimental study of effects of circular-cross-section riblets on the aerodynamic performance of Ris $\varnothing$ airfoil at transient flow regime. Journal of Mechanical Science and Technology 32(2), 709-716.

Seshagiri, A., E. Cooper and L. W. Traub (2009). Effects of vortex generators on an airfoil at low Reynolds numbers. Journal of Aircraft 46(1), 116-122.

Sreejith, B. K. and A. Sathyabhama (2018). Numerical study on effect of boundary layer trips on aerodynamic performance of E216 airfoil. Engineering Science and Technology, an International Journal 21(1), 77-88.

Tani, I. (1964). Low-speed flows involving bubble separations. Progress in Aerospace Sciences 5 , 70-103.

Tathiri, G., H. Parishani, S. G. Pouryoussefi, E Esmaeilzadeh, S. M. Mirsajedi, M. Mirzaei and N. Khatibzadeh (2016). Experimental Investigation of Separation Control on a NACA0024 Airfoil using Stationary and NonStationary AC-Dielectric Barrier Discharge Plasma Actuator. Journal of Applied Fluid Mechanics 9(2), 877-888.

Tong, F., W. Qiao, W. Chen, H. Cheng, R. Wei and X. Wang (2018). Numerical analysis of broadband noise reduction with wavy leading edge. Chinese Journal of Aeronautics 31(7), 1489-1505.

Traub, L. W. (2011). Experimental investigation of the effect of trip strips at low Reynolds number. Journal of Aircraft 48(5), 1776-1784. 
T. Durhasan / JAFM, Vol. 13, No. 6, pp. 1819-1833, 2020.

Weber, P. W., L. E. Howle, M. M. Murray and D. S. Miklosovic (2011). Computational evaluation of the performance of lifting surfaces with leading-edge protuberances. Journal of Aircraft 48(2), 591-600.

Yarusevych, S., P. E. Sullivan and J. G. Kawall (2009). On vortex shedding from an airfoil in low-Reynolds-number flows. Journal of Fluid Mechanics 632, 245-271.

Yayun, S., B. Junqiang, H. Jun and Y. Tihao (2015). Numerical analysis and optimization of boundary layer suction on airfoils. Chinese Journal of Aeronautics 28(2), 357-367.

Yousefi, K. and Saleh, R. (2014). The effects of trailing edge blowing on aerodynamic characteristics of the NACA 0012 airfoil and optimization of the blowing slot geometry. Journal of Theoretical and Applied Mechanics 52(1), 165-179
Zhang, M. M., G. F. Wang and J. Z. Xu (2013). Aerodynamic control of low-Reynolds-number airfoil with leading-edge protuberances. AIAA Journal 51(8), 1960-1971.

Zhang, W., R. Hain and C. J. Kähler (2008). Scanning PIV investigation of the laminar separation bubble on a SD7003 airfoil. Experiments in Fluids 45(4), 725-743.

Zhang, Y., H. Chen, S. Fu and W. Dong (2018). Numerical study of an airfoil with riblets installed based on large eddy simulation. Aerospace Science and Technology 78, 661670.

Zuo, Z., Q. Huang and S. Liu (2019). An Analysis on the Flow Field Structures and the Aerodynamic Noise of Airfoils with Serrated Trailing Edges Based on Embedded Large Eddy Flow Simulations. Journal of Applied Fluid Mechanics 12(2), 327-339. 